\title{
Comportamentos associados à manutenção dos resultados após cirurgia bariátrica
}

\section{Behaviours associated to maintenance of the results after bariatric surgery}

Vanessa D. S. Antonini', Luzia J. Hintze², Danilo F. da Silva ${ }^{3}$, Danielle A. M. Hermoso ${ }^{4}$, Idalina D. R. Carolino ${ }^{5}$, Nelson Nardo Jr ${ }^{6}$

\begin{abstract}
RESUMO:
A Cirurgia Bariátrica $(C B)$ é um método de tratamento da obesidade que vem recebendo destaque principalmente devido aos resultados positivos no tratamento das comorbidades associados ao excesso de peso. No entanto, esse tipo de intervenção necessita de uma fase de adaptação e mudanças de estilo de vida principalmente devido a complicações pós-cirúrgicas relacionadas à deficiência de algumas vitaminas e mineiras. Nesse sentido, o objetivo do estudo foi associar o perfil antropométrico, composição corporal e tempo desde a realização da CB com a adesão a comportamentos alimentares saudáveis de pacientes submetidos a esse procedimento. Estudo do tipo transversal realizado com 98 pacientes submetidos ao bypass gástrico em $Y$ de Roux. Foram avaliados peso, estatura, circunferência da cintura (CC) e quadril, massa de gordura, massa muscular esquelética e o comportamento alimentar por meio do questionário Bariatric Surgery Self-management Questionnaire Seven, que possui 7 domínios referentes ao comportamento alimentar (CA), à ingestão de fluidos (IF), à atividade física (AF), à gestão da síndrome de dumping (GSD), à admissão de suplementos (AS), ao consumo de frutas, vegetais e grãos integrais (CFVGI) e à ingestão de proteínas (IP). Foi verificada associação entre adesão total dos comportamentos alimentares, CFVGI, IP com o estado nutricional e CC após a CB. Observamos também menor peso, IMC e CC e menor \% e quantidade de gordura em pacientes que apresentaram boa adesão nesses domínios. Nos domínios, CA, IF e AF os pacientes com menor tempo após a realização da CB apresentaram melhor adesão a esses comportamentos. Os sujeitos mais jovens apresentaram maior adesão à $\mathrm{AF}$ comparados aos mais velhos. Em conclusão, a adesão aos comportamentos alimentares após a CB se associou com o estado nutricional e CC, bem como com o tempo após a realização à $\mathrm{CB}$. Sugere-se a realização de estudos que proponham modelos de intervenção para o melhor controle e manutenção dos comportamentos alimentares dos pacientes submetidos a esse procedimento.
\end{abstract}

Palavras-chave: Cirurgia Bariátrica. Obesidade Mórbida. Comportamento Alimentar. Perda de Peso. Estilo de Vida. Manutenção.

${ }^{1}$ Mestranda em Educação Física; ${ }^{2}$ Mestre em Educação Física; ${ }^{3}$ Doutorando em Educação Física. Programa de Pós Graduação em Educação Física Associado UEM/UEL - Universidade Estadual de Maringá/PR - Brasil.

${ }^{4}$ Mestranda Programa de Pós Graduação em Ciências Biológicas; ${ }^{5}$ Professora do Departamento de Medicina; 6Professor Associado Departamento de Educação Física. Universidade Estadual de Maringá- PR - Brasil
Correspondência: Vanessa D. S. Antonin Avenida Colombo, 5790 - Bloco M05 - Sala 4A CEP: 87020-900 - Maringá-PR / Brasil.

Artigo recebido em 27/08/2013 Aprovado para publicação em 04/02/2014 


\section{Introdução}

A obesidade tem se tornado um problema de saúde pública alcançando proporções epidêmicas e alarmantes em todo mundo. ${ }^{1,2}$ Estudos epidemiológicos mostram que a obesidade atinge $15,7 \%$ da população adulta no Brasil. Entre os homens, os valores são três vezes maiores no grupo de 35 a 44 anos (20,8\%), comparados aqueles com idade entre 18 a 24 anos $(6,3 \%)$. Para as mulheres, os valores tenderam a aumentar de 18 a 64 anos $(6,9 \%$ a $24,5 \%$ respectivamente). ${ }^{3}$ Considerando esses números, ações de prevenção e tratamento dessa patologia devem ser implementadas ${ }^{2,4}$ tendo como base mudanças no estilo de vida ${ }^{1}$. Porém, esse tipo de intervenção não é comumente disponível nos serviços de saúde, restando para a maioria das pessoas o tratamento cirúrgico da obesidade, conhecido como cirurgia bariátrica $(\mathrm{CB}) .^{5}$

Esse tipo de intervenção necessita de uma fase de adaptação e mudanças de comportamentos, de modo que, para o sucesso do tratamento, torna-se importante o acompanhamento de uma equipe multidisciplinar. ${ }^{5}$ Isso se deve ao fato de complicações no período pós-operatório serem observadas em aproximadamente $10 \%$ dos casos, como regurgitação, infecções, deiscência, estenose de estômago, tromboflebite e intolerância alimentar. ${ }^{6}$ Além disso, deficiências nutricionais se tornam comuns em pacientes que realizaram a CB e podem colocar em risco o sucesso desse tipo de intervenção. Dentre as mais comuns estão a deficiência de Vitamina $B_{12}$ e D, Cálcio, Ferro, Ácido Fólico e Tiamina. ${ }^{7,8}$ Sendo assim, a orientação nutricional específica a cada paciente em função das alterações fisiológicas e anatômicas provocadas pela cirurgia se torna necessária para identificar complicações pós-cirúrgicas, a fim de orientar e monitorar a restrição alimentar e suplementação para promover evolução da dieta evitando carências nutricionais. ${ }^{6,9}$

Pensando nisso, Welch et al. ${ }^{10}$, ao realizarem uma pesquisa referente aos comportamentos adotados por pacientes após a realização da $\mathrm{CB}$, desenvolveram um instrumento com sete domínios comportamentais, definidos a partir de uma revisão de literatura e por especialistas em CB. Os domínios são referentes a comportamento alimentar (CA), ingestão de líquidos, ingestão de proteínas, atividade física, controle da Síndrome de Dumping, ingestão de frutas, grãos integrais e vegetais e ingestão de suplementos minerais e vitamínicos. Foi observada uma grande variação de aderência aos comportamentos (61 a 90\%) no período de seis meses após a $\mathrm{CB}$, com maior aderência ao uso de suplementos e menor a prática de Atividade Física (AF). Após 18 meses, foi observada redução da aderência em quase todos os domínios, exceto a AF e consumo de frutas, vegetais e grãos integrais.

Apesar dos estudos que apontam que a CB pode ser eficaz no tratamento de comorbidades associadas à obesidade ${ }^{11,12}$, no Brasil não há trabalhos que investigaram comportamentos após a CB (CACB), sobretudo, os relacionados ao consumo de suplementos vitamínicos, de alimentos integrais, de proteínas, de frutas e hortaliças e de alimentos que podem desencadear a Síndrome de Dumping e possíveis complicações. Ademais, até onde temos conhecimento, não se sabe se a adesão aos CACB estaria associada ao perfil antropométrico e composição corporal dos pacientes antes e depois da realização da cirurgia.

Desse modo, o presente estudo objetivou associar o perfil antropométrico, composição corporal e tempo desde a realização da CB com a adesão aos $\mathrm{CACB}$ de pacientes submetidos a esse procedimento. A hipótese do estudo foi que haveria associação entre o perfil antropométrico, composição corporal e o tempo desde a realização da $\mathrm{CB}$ com os $\mathrm{CACB}$ dos pacientes avaliados.

\section{Materiais e Métodos}

\section{Participantes}

Participaram do estudo 98 pacientes submetidos à CB pelo Sistema Único de Saúde (SUS) com idade entre 20 e 74 anos que realizaram a CB entre 2000 e 2010. Todos os pacientes foram contatados por telefone e convidados a participar do estudo. Aqueles que compareceram a reunião previamente agendada foram esclarecidos quanto às suas dúvidas e todos os que concordaram, assinaram o termo de consentimento livre e esclarecido. O estudo foi aprovado pelo Comitê de Ética local (parecer 412/2008).

\section{Avaliações}

Foi medido o peso corporal utilizando uma balança eletrônica da marca Welmy, com capacidade de medida de $300 \mathrm{~kg}$ e precisão de 50 g. Para mensuração da estatura utilizou-se um estadiômetro acoplado a parede com precisão de $0,1 \mathrm{~cm}$ da marca Sanny seguindo as orientações propostas por Heyward e Stolarczyk (2000). O Índice de Massa Corporal (IMC) foi calculado com base na divisão do peso pela estatu- 
ra ao quadrado. A circunferência da cintura (CC) foi determinada conforme padronização do WHO (1995), fazendo uso de uma fita antropométrica com capacidade de medida de $2 \mathrm{~m}$ e precisão de $0,1 \mathrm{~cm}$.

As medidas da composição corporal foram feitas pelo método da absorciometria por raios $\mathrm{X}$ de dupla energia (DXA) da marca GE modelo prodigy pri$m o$; foi verificado o percentual de gordura corporal (\% MG), a massa magra (MM) a massa de gordura corporal (MG).

Para cálculo do peso ideal e Excesso de Peso Perdido (EPP) foram utilizadas as seguintes equações:

Equação 1: \% EPP= (Peso Pré CB- Peso Atual $)$

(Peso Pré CB - Peso ideal) x 100

Equação 2: Peso Ideal $=$ estatura $^{2} \times 25$

Considerando $25 \mathrm{~kg} / \mathrm{m}^{2}$ para o IMC como o valor superior ao limite da normalidade.

Para avaliar os CACB foi utilizado o questionário Bariatric Surgery Self-management Questionnaire Seven. Após uma revisão de literatura e entrevistas por profissionais da área (medicina comportamental, médicos, enfermeira e nutricionista) os autores do instrumento identificaram sete domínios com seus conteúdos desenvolvidos por um processo de consenso entre a equipe, com o objetivo de criar itens que representassem suficientemente cada domínio. Os itens avaliam a autogestão dos comportamentos realizados pelos pacientes sendo esperadas elevadas taxas de adesão para aumentar o sucesso após o procedimento. ${ }^{10}$

Os domínios são: comportamento alimentar (CA), ingestão de fluidos (IF), atividade física (AF), gestão da síndrome de dumping (GSD), admissão de suplementos (AS), consumo de frutas, vegetais e grãos integrais (CFVGI) e ingestão de proteínas (IP). No questionário, há questões referentes a esses domínios com duas alternativas de resposta - sim (representa bons comportamentos) e não (maus comportamentos). Um escore de adesão foi calculado a partir das respostas positivas de cada domínio do instrumento, sendo que todas as respostas positivas representam $100 \%$ de aderência ao domínio. ${ }^{10}$ Foram estabelecidos para as análises valores superiores a $60 \%$ como boa adesão.

Todos estes procedimentos foram realizados no laboratório equipado para tal, sendo que os equipamentos foram manipulados por profissionais e/ou estudantes devidamente qualificados para a realização de tais avaliações.

\section{Análise Estatística}

A normalidade dos dados foi testada a partir do teste de Shapiro-Wilk. Com base nesses resultados, utilizou-se para a análise descritiva mediana e amplitude interquartílica. A análise inferencial foi feita por meio do teste de associação Qui-quadrado $2 \times 2$ para a associação entre o estado nutricional (obeso (IMC $\geq$ $\left.30 \mathrm{~kg} / \mathrm{m}^{2}\right)$ e não obeso $\left(\mathrm{IMC}<30 \mathrm{~kg} / \mathrm{m}^{2}\right)$ ) e obesidade abdominal (com CC normal $(<102 \mathrm{~cm}$ para homens e $<88 \mathrm{~cm}$ para mulheres) e com CC aumentada ( $\geq 102$ $\mathrm{cm}$ para homens e $\geq 88 \mathrm{~cm}$ para mulheres) com os CACB. Além disso, as variáveis, idade, tempo desde a realização da $\mathrm{CB}$, peso antes da $\mathrm{CB}$, IMC antes da $\mathrm{CB}$, peso atual, IMC atual e $\mathrm{CC}$ foram comparadas de acordo com a adesão ou não dos CACB através do teste não-paramétrico de Mann-Whitney. A significância estatística foi pré-estabelecida em $\mathrm{p}<0,05$.

\section{Resultados}

Os pacientes incluídos no presente estudo apresentaram média de idade de $49 \pm 12$ anos. Em relação ao sexo, a maioria dos pacientes que realizaram a $\mathrm{CB}$ foi de mulheres $(87 \%, \mathrm{n}=86)$.

Na tabela 1 estão apresentados os resultados da associação da adesão dos CACB com o estado nutricional (obeso e não obeso) e a obesidade abdominal (CC normal e aumentada). Os resultados demonstraram que uma boa adesão aos CACB, de um modo geral (adesão total), esteve associada a um IMC menor que $30 \mathrm{~kg} / \mathrm{m}^{2}$ (não obeso) como um CC normal dos indivíduos avaliados. Além disso, a boa adesão a comportamentos específicos (CFVGI e IP) esteve associada a CC normal.

As tabelas 2 e 3 apresentam os resultados da comparação dos grupos de boa e má adesão aos domínios CFVGI e IP para as variáveis antropométricas e de composição corporal. Foram verificadas que os sujeitos que mais aderiram a esses comportamentos apresentam melhores resultados em relação ao peso e IMC atuais, \%EPP, CC e gordura absoluta e relativa.

Os demais CACB, de uma maneira geral, foram aderidos pelos pacientes com menor tempo cirúrgico, especialmente no que diz respeito aos domínios $\mathrm{CA}(\mathrm{p}=0,002), \operatorname{IF}(\mathrm{p}=0,004)$ e AF $(\mathrm{p}=0,001)$. Ainda para o domínio da AF, os pacientes mais jovens (40 (15) anos) aderiam mais a este comportamento comparado aos mais velhos (54 (16) anos) ( $\mathrm{p}=<0,001)$. Em relação aos domínios GSD e AS não foram 
Tabela 1: Associação entre estado nutricional, obesidade abdominal e CA de pacientes submetidos à $\mathbf{C B}(\mathbf{n}=98)$.

\begin{tabular}{|c|c|c|c|c|c|c|}
\hline & Não Obeso & Obeso & $\mathbf{P}$ & CC normal & CCaumentado & $\mathbf{P}$ \\
\hline \multicolumn{7}{|c|}{ Adesão do CA } \\
\hline Sim & 32 & 24 & 0,162 & 28 & 28 & 0,483 \\
\hline Não & 18 & 24 & & 18 & 24 & \\
\hline \multicolumn{7}{|c|}{ Adesão do IF } \\
\hline Sim & 25 & 23 & 0,837 & 21 & 27 & 0,535 \\
\hline Não & 25 & 25 & & 25 & 25 & \\
\hline \multicolumn{7}{|c|}{ Adesão daAF } \\
\hline Sim & 12 & 7 & 0,238 & 12 & 7 & 0,115 \\
\hline Não & 38 & 41 & & 34 & 45 & \\
\hline \multicolumn{7}{|c|}{ Adesão da GSD } \\
\hline Sim & 16 & 9 & 0,133 & 13 & 12 & 0,557 \\
\hline Não & 34 & 39 & & 33 & 40 & \\
\hline \multicolumn{7}{|c|}{ Adesão de AS } \\
\hline Sim & 7 & 6 & 0,827 & 8 & 5 & 0,257 \\
\hline Não & 43 & 42 & & 38 & 47 & \\
\hline \multicolumn{7}{|c|}{ Adesãodo CFVGI } \\
\hline Sim & 36 & 23 & $0,015^{*}$ & 34 & 25 & $0,009 *$ \\
\hline Não & 14 & 25 & & 12 & 27 & \\
\hline \multicolumn{7}{|c|}{ Adesão de IP } \\
\hline Sim & 33 & 16 & $0,001 *$ & 31 & 18 & $0,001 *$ \\
\hline Não & 17 & 32 & & 15 & 34 & \\
\hline \multicolumn{7}{|c|}{ Adesão total } \\
\hline Sim & 16 & 7 & $0,042^{*}$ & 13 & 10 & 0,292 \\
\hline Não & 34 & 41 & & 33 & 42 & \\
\hline
\end{tabular}

${ }^{*} \mathrm{p}<0,05$.

$\mathrm{CA}=$ comportamento alimentar; $\mathrm{CB}=$ Cirurgia Bariátrica; $\mathrm{CC}=$ circunferência da cintura.

Tabela 2: Comparação da idade, resultados da CB, perfil antropométrico e composição corporal de pacientes que após a CB aderiram e não aderiram aos CFVGI.

\begin{tabular}{lccccl}
\hline & Adesão $(\mathbf{n}=\mathbf{5 9})$ & Me & Má Adesão $(\mathbf{n}=\mathbf{3 9})$ & $\mathbf{a}$ & $\mathbf{P}$ \\
\hline Idade (anos) & $\mathbf{M e}$ & $\mathbf{a Q}$ & $\mathbf{M e}$ & $\mathbf{a Q}$ \\
Tempo CB (meses) & 49 & 16 & 54 & 19 & 0,193 \\
Peso pré $(\mathrm{kg})$ & 45 & 71 & 88 & 73 & $0,022^{*}$ \\
IMC pré $\left(\mathrm{kg} / \mathrm{m}^{2}\right)$ & 118 & 26 & 124 & 33 & 0,250 \\
Peso atual $(\mathrm{kg})$ & 47 & 11 & 50 & 9 & 0,230 \\
IMC atual $\left(\mathrm{kg} / \mathrm{m}^{2}\right)$ & 74 & 20 & 85 & 25 & $0,022^{*}$ \\
\%EPP & 29 & 9 & 34 & 11 & $0,008^{*}$ \\
CC $(\mathrm{cm})$ & 78 & 26 & 60 & 33 & $0,003^{*}$ \\
Gordura corporal $(\%)$ & 86 & 17 & 95 & 25 & $0,016^{*}$ \\
Gordura corporal $(\mathrm{kg})$ & 42 & 12 & 48 & 13 & $0,012^{*}$ \\
Massa Magra $(\mathrm{kg})$ & 28 & 18 & 41 & 19 & $0,008^{*}$ \\
\hline
\end{tabular}

${ }^{*} \mathrm{p}<0,05$. 
verificadas diferenças em relação ao tempo cirúrgico, variáveis antropométricas e de composição corporal, quando comparados os pacientes de boa e má adesão.

A análise de adesão total (todos os sete domínios) está apresentada na tabela 4. Quando considerados os sete domínios do CACB, observou-se que um menor tempo de cirurgia foi determinante para uma maior adesão, bem como um menor IMC atual.

\section{Discussão}

O objetivo do presente estudo foi associar o perfil antropométrico, composição corporal e tempo desde a realização da $\mathrm{CB}$ com a adesão aos CACB de pacientes submetidos a esse procedimento. O principal achado do presente estudo é a baixa adesão aos comportamentos recomendados para a manutenção

Tabela 3: Comparação da idade, resultados da CB, perfil antropométrico e composição corporal de pacientes que após a CB aderiram e não aderiram à recomendação de IP.

\begin{tabular}{|c|c|c|c|c|c|}
\hline & \multicolumn{2}{|c|}{ Adesão (n=49) } & \multicolumn{3}{|c|}{ Má Adesão (n=49) } \\
\hline & Me & $\mathbf{a}_{\mathrm{Q}}$ & Me & $\mathbf{a}_{\mathbf{Q}}$ & $\mathbf{P}$ \\
\hline Idade (anos) & 49 & 18 & 54 & 15 & 0,215 \\
\hline Tempo CB (meses) & 39 & 29 & 96 & 63 & $<0,001 *$ \\
\hline Peso pré (kg) & 115 & 24 & 128 & 27 & $0,007^{*}$ \\
\hline IMC pré $\left(\mathrm{kg} / \mathrm{m}^{2}\right)$ & 45 & 11 & 50 & 9 & $0,012^{*}$ \\
\hline Peso atual (kg) & 73 & 19 & 84 & 24 & $0,007^{*}$ \\
\hline IMC atual $\left(\mathrm{kg} / \mathrm{m}^{2}\right)$ & 28 & 8 & 34 & 10 & $0,002 *$ \\
\hline$\%$ ЕPP & 80 & 29 & 67 & 35 & $0,003^{*}$ \\
\hline $\mathrm{CC}(\mathrm{cm})$ & 85 & 18 & 94 & 25 & $0,003^{*}$ \\
\hline Gordura corporal (\%) & 41 & 13 & 48 & 12 & $0,035^{*}$ \\
\hline Gordura corporal (kg) & 27 & 19 & 40 & 19 & $0,008^{*}$ \\
\hline Massa Magra (kg) & 40 & 8 & 42 & 9 & 0,122 \\
\hline
\end{tabular}
${ }^{*} \mathrm{p}<0,05$.

Tabela 4: Comparação da idade, resultados da CB, perfil antropométrico e composição corporal de pacientes que após a CB aderiram e não aderiram ao somatório de comportamentos (total).

\begin{tabular}{|c|c|c|c|c|c|}
\hline \multicolumn{3}{|c|}{ Adesão $(n=23)$} & \multicolumn{3}{|c|}{ Má Adesão (n=75) } \\
\hline & Me & $\mathbf{a}_{\mathbf{Q}}$ & Me & $\mathbf{a}_{\mathbf{Q}}$ & $\mathbf{P}$ \\
\hline Idade (anos) & 49 & 18 & 51 & 18 & 0,642 \\
\hline Tempo CB (meses) & 34 & 35 & 79 & 69 & $0,001^{*}$ \\
\hline Peso pré (kg) & 115 & 32 & 122 & 29 & 0,263 \\
\hline IMC pré $\left(\mathrm{kg} / \mathrm{m}^{2}\right)$ & 46 & 11 & 49 & 10 & 0,190 \\
\hline Peso atual (kg) & 74 & 18 & 82 & 29 & 0,148 \\
\hline IMC atual $\left(\mathrm{kg} / \mathrm{m}^{2}\right)$ & 29 & 8 & 32 & 10 & $0,045^{*}$ \\
\hline$\%$ EPP & 79 & 30 & 70 & 39 & 0,075 \\
\hline $\mathrm{CC}(\mathrm{cm})$ & 86 & 11 & 93 & 24 & 0,054 \\
\hline Gordura corporal (\%) & 42 & 12 & 47 & 14 & 0,176 \\
\hline Gordura corporal (kg) & 29 & 17 & 35 & 21 & 0,115 \\
\hline Massa Magra (kg) & 41 & 8 & 41 & 9 & 0,610 \\
\hline
\end{tabular}

${ }^{*} p<0,05$. 
dos resultados da $\mathrm{CB}$, somente um quarto dos pacientes aderiu aos 7 compartamentos avaliados. Além disso, verificou-se associação entre adesão total dos CACB, CFVGI, IP com o estado nutricional e CC após a CB. Observamos também diferenças nas variáveis antropométricas (menor peso, IMC e CC) e de composição corporal (menor \% e kg de gordura) em pacientes que apresentaram boa adesão nesses domínios. Nos domínios, CA, IF e AF os pacientes com menor tempo após a realização da $\mathrm{CB}$ apresentaram melhor adesão a esses comportamentos. Os sujeitos mais jovens apresentaram maior adesão à $\mathrm{AF}$ comparados aos mais velhos.

Um dos resultados encontrados no estudo refere-se ao melhor perfil de composição corporal e variáveis antropométricas após a $\mathrm{CB}$ dos pacientes que tiveram boa adesão ao CFVGI. O baixo consumo de frutas, verduras e legumes, segundo a $\mathrm{WHO}^{13}$ está entre os dez principais fatores de risco para doenças em todo o mundo. O seu consumo, além de possibilitar o atendimento ou a obtenção das recomendações diárias de consumo de vitaminas e minerais, em função do papel desses nutrientes na prevenção de doenças não transmissíveis ${ }^{14}$, tem baixa densidade energética e alto teor de fibras o que promove maior saciedade favorecendo a perda de peso e manutenção do mesmo ${ }^{15}$. Esses achados podem explicar os melhores resultados dos pacientes que apresentaram boa adesão a esse comportamento além de maior \%EPP.

Em relação à IP, a Associação Americana de Endocrinologistas Clínicos e a Sociedade Americana de Cirurgia Bariátrica e Metabólica ${ }^{16}$ apontaram que $17 \%$ dos pacientes submetidos à $\mathrm{CB}$ vivenciam intolerância alimentar de fontes proteicas, o que pode limitar a ingestão em $50 \%$ do total recomendado. No entanto, esses problemas tendem a diminuir a partir de um ano de cirurgia.

Há evidências demonstrando que o aumento no consumo de proteínas em longo prazo, independentemente da sua origem, auxilia na manutenção do peso perdido após sua perda induzida por restrição dietética por contribuir na preservação da massa magra. Esses benefícios podem ser decorrentes de uma maior capacidade de saciedade das proteínas em relação aos carboidratos e um maior efeito térmico desses alimentos. ${ }^{17,18}$ No presente estudo, a boa adesão a IP esteve associada à quantidade e distribuição de gordura, mas não a massa magra.

As recomendações proteicas específicas durante dietas restritas ainda não estão bem definidas e podem variar de 60 a $80 \mathrm{~g} /$ dia ou 1,0 a $1,5 \mathrm{~g} / \mathrm{kg}$ de peso ideal. Normalmente, para atender essas orientações, o paciente pode utilizar suplementos proteicos em pó de alto valor biológico. ${ }^{19,20}$

Outra variável que parece ter relação com uma maior adesão aos CACB é o tempo após a realização da $\mathrm{CB}$, em que os pacientes que operaram há menos tempo também apresentaram maior adesão ao CA, IF e AF. Esse resultado corrobora com os achados de Freire et al. ${ }^{21}$, em que foi verificado que a má qualidade da dieta, sedentarismo e falha em acompanhamento nutricional em longo prazo foram os principais fatores associados à falha na manutenção do peso perdido após 5 anos de CB. A dieta desses pacientes operados há mais tempo foi caracterizada por excesso de ingestão de calorias, alimentos gordurosos, açúcares e lanches. Isso pode ser explicado por alterações nos níveis de hormônios que regulam o consumo alimentar e redução na atividade física após a CB. ${ }^{22}$

Considerando os achados do presente estudo, o procedimento cirúrgico para a redução do excesso de peso não pode ser visto como a resposta final ao problema da obesidade. Ao contrário, como destacam os autores da rede canadense de obesidade (Canadian Obesity Network - CON) em seu algoritmo para avaliação e tratamento de pessoas em condições de sobrepeso e obesidade, a intervenção multiprofissional deve ser oferecida nos casos em que a $\mathrm{CB}$ não promover os resultados esperados. ${ }^{1}$ Considerando o impacto do tempo da CB sobre a redução da adesão ao $\mathrm{CA}$, IF e AF, a promoção de estratégias para manutenção dos CACB em longo prazo deve ser estimulada, para que não haja novo ganho da gordura corporal, IMC e CC que podem estar associados à presença de fatores de risco cardiovasculares. ${ }^{23,24}$

A idade teve impacto sobre a AF após a CB, sendo que os pacientes mais jovens apresentaram maior adesão comparada aos mais velhos. Considerando as dificuldades que o avanço na idade naturalmente traz para a prática regular de atividade física, e a falta de um acompanhamento por um profissional de educação física após a realização da CB, é fundamental que estratégias para estimular a prática regular de exercícios físicos sejam incentivadas, tais como a integração de profissionais de educação física às equipes multiprofissionais em hospitais responsáveis pelo acompanhamento de indivíduos submetidos à CB. ${ }^{10,21,25}$ Uma das possibilidades seria a criação de equipes multidisciplinares, incluindo profissionais das áreas da nutrição, psicologia, medicina e educação fí- 
sica a fim de monitorar e aconselhar as mudanças no comportamento alimentar e de atividade física que são necessárias para manutenção dos resultados obtidos com a cirurgia.

Apesar das contribuições, o presente estudo também apresenta algumas limitações. Foram avaliados pacientes no período pós-cirúrgico e foram utilizados dados auto referidos relativos as condições dos indivíduos no momento pré cirúrgico. Embora o objetivo do presente estudo seja o de associar os comportamentos alimentares aos resultados obtidos com a CB, a mensuração de parâmetros antropométricos, composição corporal e comportamento alimentar com frequência anual, após a intervenção cirúrgica, poderia confirmar ou refutar os resultados aqui encontrados. Outra questão refere-se à seleção da amostra, a qual foi feita por conveniência, sendo incluídos no estudo os pacientes que apresentaram interesse em participar da pesquisa, residentes na cidade de Maringá-PR e região. Possivelmente, pacientes não interessados em participar da pesquisa podem apresentar resultados diferentes dos encontrados no presente estudo. Porém, cabe ressaltar que buscamos a amostra mais representativa possível de pacientes do SUS.
Em conclusão, a adesão aos CACB foi baixa e se associou com o estado nutricional e CC após a CB, bem como com o tempo após a realização à $\mathrm{CB}$. Sugere-se a realização de estudos que proponham modelos de intervenção para o melhor controle e manutenção dos CACB dos pacientes submetidos a esse procedimento.

\section{Agradecimentos}

Agradecemos a todos os pacientes que concordaram em participar deste estudo. Agradecemos aos demais membros do Núcleo de Estudos Multiprofissional da Obesidade pela contribuição nas coletas de dados. Agradecemos a Financiadora de Estudos e Projetos (FINEP) pelo financiamento desta pesquisa e a Coordenação de Aperfeiçoamento de Pessoal de Nível Superior (CAPES) pelas bolsas de estudo proporcionadas.

\section{Suporte Financeiro}

Financiadora de estudos e Projetos - FINEP e Coordenação de Aperfeiçoamento de Pessoal do Ensino Superior - CAPES pelas bolsas de estudos proporcionadas.

\begin{abstract}
:
Bariatric Surgery (BS) is a method for obesity treatment which has been receiving attention mainly due to the positive results in the treatment of comorbidities associated with overweight. However, this type of intervention requires a period of adaptation and change of lifestyle mainly due to post surgery complications related to vitamins and minerals deficits. In this way, the objective of this study was to associate the anthropometric profile, body composition and time since the surgery with the adherence to healthy behavior eating on patients who underwent this procedure. A cross-sectional study was conducted with 98 patients who underwent gastric bypass Roux-Y. We evaluated weight, height, waist (WC) and hip circumferences, body fat (BF), skeletal muscle mass and feeding behavior by the Bariatric Surgery Selfmanagement Questionnaire Seven, which has 7 domains related to feeding behavior (FB), the fluid intake (FI), physical activity (PA), management of Dumping Syndrome (MDS), admission of supplements (AS), consume of fruits, vegetables and whole grains (CFVWG) and protein intake (PI). We verify association between total adherence of eating behaviors, CFVWG, and PI with nutritional status and WC after BS. We also observed lower weight, BMI and WC, \% and quantity of BF in patients who presented good adherence in these domains. In the domains FB, FI, and PA the patients who had operated in less time present better adherence. The younger subjects showed higher PA adhesion compared to the older ones. In conclusion, feeding behaviors adherence after BS was associated with nutritional status and WC, as well as time since BS. It is suggested to carry out studies that propose intervention models for the better control and maintenance of feeding behaviors in patients who underwent BS.
\end{abstract}

Key-words: Bariatric Surgery. Obesity, Morbid. Feeding Behavior. Weight Loss. Life Style. Maintenance. 


\section{Referências}

1. Lau DCW, Douketis JD, Morrison KM, Hramiak IM, Sharma AM, Ur E. Canadian clinical practice guidelines on the management and prevention of obesity in adults and children. CMAJ 2007; 176: 8.

2. World Health Organization. European ministerial conference on counteracting obesity: diet and physical activity for health. Istambul; 2006

3. Vigitel. Vigilância de fatores de risco e proteção para doenças crônicas por inquérito telefônico. Estimativas sobre frequência e distribuição sociodemográfica de fatores de risco e proteção para doenças crônicas nas capitais dos 26 estados brasileiros e no distrito federal em 2011. Brasília, DF, 2012.

4. Centers for Disease Control And Prevention. Participation in High School Physical Education: United States 1991-2003. MMWR: Morbidity and mortality weekly report, Atlanta 2004; 53: 844-7.

5. BRASIL. Conselho Nacional de Secretários de Saúde. Assistência de Média e Alta Complexidade no SUS/ Conselho Nacional de Secretários de Saúde. - Brasília :. CDD - 20. ed. - 362.1068. Conass, 2007.

6. Quadros MRR, Savaris AL, Ferreira, M. V.; Branco Filho, A.J. Intolerância alimentar no pós-operatório de pacientes submetidos à cirurgia bariátrica. Rev Bras Nutr Clin. 2007; 22: 15-9.

7. Soares CC, Falcão NC. Abordagem nutricional nos diferentes tipos de cirurgia bariátrica. Rev Bras Nutr Clin. 2007; 22: 55-64.

8. Xanthakos AS, Inge TH. Nutritional consequences of bariatric surgery. Curr Opin Clin Nutr Metab Care. 2006; 9: 489-96.

9. Cruz MRR, Morimoto IMI. Intervenção nutricional no tratamento cirúrgico da obesidade mórbida:resultados de um protocolo diferenciado. Rev Nutr PUCCAMP. 2004; 17: 263-77.

10. Welch G, Wesolowski C, Piepul B, Kuhn J, Romanelli J, Garb J. Physical Activity Predicts Weight Loss Following Gastric Bypass Surgery: Findings from a Support Group Survey. Obes Surg. 2008; 18: 517-24.

11. Kruseman M, Leimgruber A, Zumbach F, Golay A. Dietary, weight, and psychological changes among patients with obesity, 8 years after gastric bypass. J Am Diet Assoc. 2010; 110:527-34

12. Naef M, Mouton WG, Naef U, Kummer O, Muggli B, Wagner HE. Graft survival and complications after laparoscopic gastric banding for morbid obesity-lessons learned from a 12year experience. Obes Surg. 2010; 20:1206-14.
13. World Health Organization, The World Health Report 2002: Reducing Risks, Promoting Healthy Life. Geneva, Switzerland, 2002: 248.

14. Mahan LK, Escott-Stump S. KRAUSE- Alimentos, Nutrição \& Dietoterapia. São Paulo, Roca, 2005.

15. Van Duyn MA, Pivonka E. Overview of the health benefits of fruit and vegetable consumption for the dietetics professional: selected literature. J Am Diet Assoc. 2000; 100:151121.

16. AACE/TOS/ASMBS Guidelines. Perioperative Bariatric Guidelines. Obes J. 2009; Suppl 1: 17.

17. Dâmaso A. Nutrição e Exercício na Prevenção de Doenças. 2ed. Rio de Janeiro: Guanabara Koogan, 2012.

18. Westerterp-Platenga MS, Nieuwenhuizen A, Tomé D, Soenen S, Westerterp KR. Dietary Protein,Weight Loss, and Weight Maintenance. Annu Rev Nutr. 2009; 29: 21-41.

19. Skroubis G, Anesidis S, Kehagias I, Mead N, Vagenas K, Kalfarentzos F. Roux-en-Y gastric bypass versus a variant of biliopancreatic diversion in a non-superobese population: prospective comparison of the efficacy and the incidence of metabolic deficiencies. Obes Surg. 2006; 16:488-95.

20. Brolin R, Lamarca L, Kenler H, Cody R. Malabsorptive gastric bypass in patients with superobesity. J Gastrointest Surg. 2002; 6:195-205.

21. Freire RH, Borges MC, Alvarez-Leite JI, Toulson Davisson Correia Ml. Food quality, physical activity, and nutritional follow up as determinant of weight regain after Roux-en-Y gastricbypass. Nutrition. 2012; 28:53-8.

22- Shah M, Simha V, Garg A. Review: Long-term impact of bariatric surgery on body weight, comorbidities, and nutritional status. J Clin Endocrinol Metab. 2006; 91:4223-31.

23. Du SM et al. Relationship of Body Mass Index, Waist Circumference and Cardiovascular Risk Factors in Chinese Adult. Biomed Environ Sci. 2010; 23: 92-101.

24. Sarno F, Monteiro CA. Importância relativa do Índice de Massa Corporal e da circunferência abdominal na predição da hipertensão arterial. Rev Saúde Pública. 2007; 41:788-96.

25. Jacobi D, Ciangura C, Couet C, Oppert JM. Physical activity and weight loss following bariatric surgery. Obes Rev. 2011; 12:366-77. 